\title{
Qualitätsmerkmale von RFID Etiketten
}

\author{
Josef Bernhard, Tobias Dräger
}

Um die Qualität eines RFID Etiketts objektiv und reproduzierbar bewerten zu können sind entsprechende Qualitätsmerkmale und Prüfverfahren notwendig. In diesem Beitrag wird kurz auf Qualitätsmerkmale im Bezug auf RFID Etiketten eingegangen und die aktuelle Situation bei der Definition von Testkriterien und- verfahren dargestellt.

\section{Motivation}

Für die Performance eines RFID Systems ist nicht nur der RFID Leser oder Reader wichtig, sondern auch die Qualität der RFID Etiketten oder Transponder entscheidend. Da die RFID Transponder, die an den Medien angebracht werden, in großen Mengen verwendet werden, ist der Preis des RFID Etiketts ein wichtiger Faktor. Getrieben durch Anwendungen im Bereich der Konsumgüterindustrie sind die Preise für RFID Etiketten insbesondere der UHF-Technologie, aber auch der HF-Technologie in den letzten Jahren massiv gesunken. Damit sich dieser Preisverfall nicht auf die Qualität niederschlägt, ist es wichtig, die Qualität der Labels von Zeit zu Zeit zu überprüfen und objektiv zu bewerten. Dieser Beitrag widmet sich dem Thema Qualitätsmerkmale bei RFID Transpondern, insbesondere der RFID Etiketten im Frequenzbereich 13,56 MHz, die derzeit fast ausschließlich in Bibliotheken zum Einsatz kommen. Es soll ein Überblick gegeben werden, welche Merkmale zur Beurteilung eines RFID Transponders herangezogen werden können und welche Normen bereits existieren.

\section{Merkmale zur Qualitätsbeurteilung}

\section{Elektrische Eigenschaften}

Als elektrische Eigenschaften werden hier die auf die Luftschnittstelle bezogenen Kennwerte eines RFID Systems verstanden. Für den Anwender ist das im Wesentlichen die Lesereichweite, die mit einem bestimmten RFID System, bestehend aus einem RFID Reader und einem RFID Transponder, erreicht werden kann. Hat der Anwender eine bestimmte Infrastruktur, sprich RFID Reader installiert, möchte er möglichst mit Etiketten unterschiedlicher Hersteller eine gleich gute Reichweite erzielen. Die Labelhersteller wiederum werden versuchen, eine möglichst gleich gute Qualität bei unterschiedlichen Readern zu erzielen. Für eine zuverlässige Funktion in der Praxis wird beispielsweise eine Lesereichweite am Selbstverbucher von etwa $35 \mathrm{~cm}$ erwartet, wenn der RFID Transponder am Medium angebracht ist. Die Lesereichweite eines Transponders an einem bestimmten, verbauten Leser zu bestimmen ist zwar keine objektiv ausreichende Messung, liefert aber für den Anwender in der Praxis eine gute Basis. Aus wissenschaftlicher Sicht 
ist diese Methode allerdings fehlerbehaftet, weil die Testumgebung nicht spezifiziert ist und damit die Messung insbesondere durch Einflüsse:

- unterschiedlicher Reader

- der Umgebung des Readers

- des Mediums

- unterschiedlicher Positionierung der Medien

verfälscht wird.

Die Reproduzierbarkeit der einzelnen Messungen und der Vergleichbarkeit der Messungen untereinander ist dadurch schwierig. Für eine objektive Charakterisierung muss also die Messumgebung klar definiert sein.

Andere relevante elektrische Leistungseigenschaften sind die Datenerhaltungszeit der gespeicherten Daten auf dem Chip und die Pulkerfassungsmöglichkeiten, also die gleichzeitige Erfassung von mehreren Transpondern.

\section{Mechanische Eigenschaften}

$\mathrm{Zu}$ den mechanischen Eigenschaften zählen Eigenschaften wie:

- Festigkeit und Langlebigkeit der Klebeverbindung

- Beständigkeit der Labeloberfläche

- Beständigkeit der Aufbau- und Verbindungstechnik des Etiketts (z. B. Antenne-Chip Verbindung)

Insbesondere für die mechanischen Eigenschaften sind Alterungstests zu definieren, aus denen zuverlässige Aussagen über die langfristige Beständigkeit der mechanischen Festigkeit des Labels abgeleitet werden können. Die mechanischen Eigenschaften zusammen mit der Erhaltungszeit der Daten auf dem Speicher des Mikrochips bestimmen die Nutzungsdauer des RFID Etiketts. Dabei ist die Gesamtlebensdauer nur so gut wie das schwächste Glied in der Kette.

\section{Zur Funktion des RFID Systems}

Zur Charakterisierung der RFID Technologie, insbesondere einzelner Komponenten eines RFID Systems, ist eine kurze Beschreibung der Technologie erforderlich, die sich im Folgenden auf die HF-Technologie bei 13,56 MHz beschränken soll.

Ein RFID System bei 13,56 MHz kann als einfacher Transformator beschrieben werden, bei dem eine Primärspule, in diesem Fall des Readers, mit einer Sekundärspule im Transponder über das magnetische Feld verkoppelt ist. 


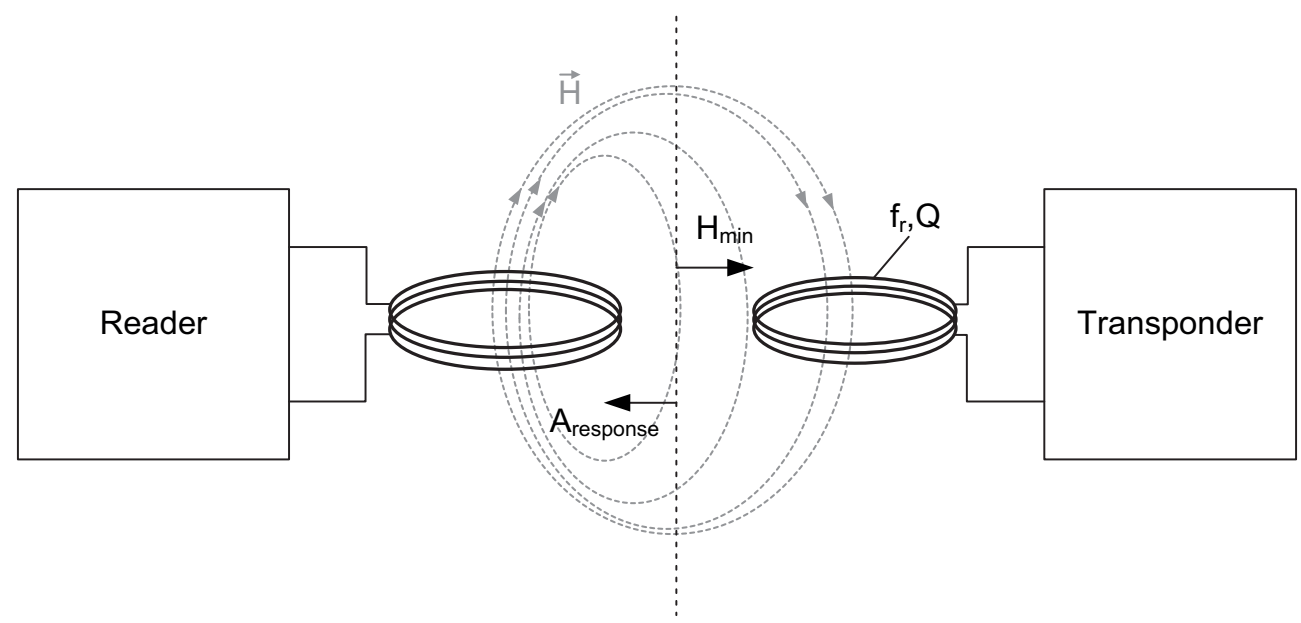

Abb. 1: RFID System mit gekoppelten Spulen

Über dieses verkoppelte Spulensystem wird einerseits Energie vom Reader an den Transponder übertragen und anderseits Information zwischen den beiden Komponenten ausgetauscht. Die Sekundärspule ist mit der Transponderelektronik verbunden, ein elektronischer Schaltkreis, der mit Energie versorgt werden muss. Da eine Batterie oder Akku für die meisten RFID Anwendungen zu teuer wäre, muss die Energie über das Feld der verkoppelten Spulen von der Primärseite - also dem Reader - zur Sekundärseite übertragen werden. Je besser die Spulen miteinander verkoppelt sind, desto mehr Energie kann übertragen werden.

Ein Maß für die Verkopplung der beiden Spulen ist der Koppelfaktor k. Es gibt verschiedene Faktoren, die den Koppelfaktor zwischen Reader- und Transponderspule beeinflussen:

- Die Geometrie, Windungszahl und Größen der Spulen

- Die Distanz zwischen Primär- und Sekundärspule

- Die Umgebung der Spulenantennen

Der Graph in der Abbildung zeigt qualitativ, wie stark der Koppelfaktor über den Abstand abfällt. Der Abstand ist in der Grafik normiert auf den Spulenradius r1 der Primärspule. 


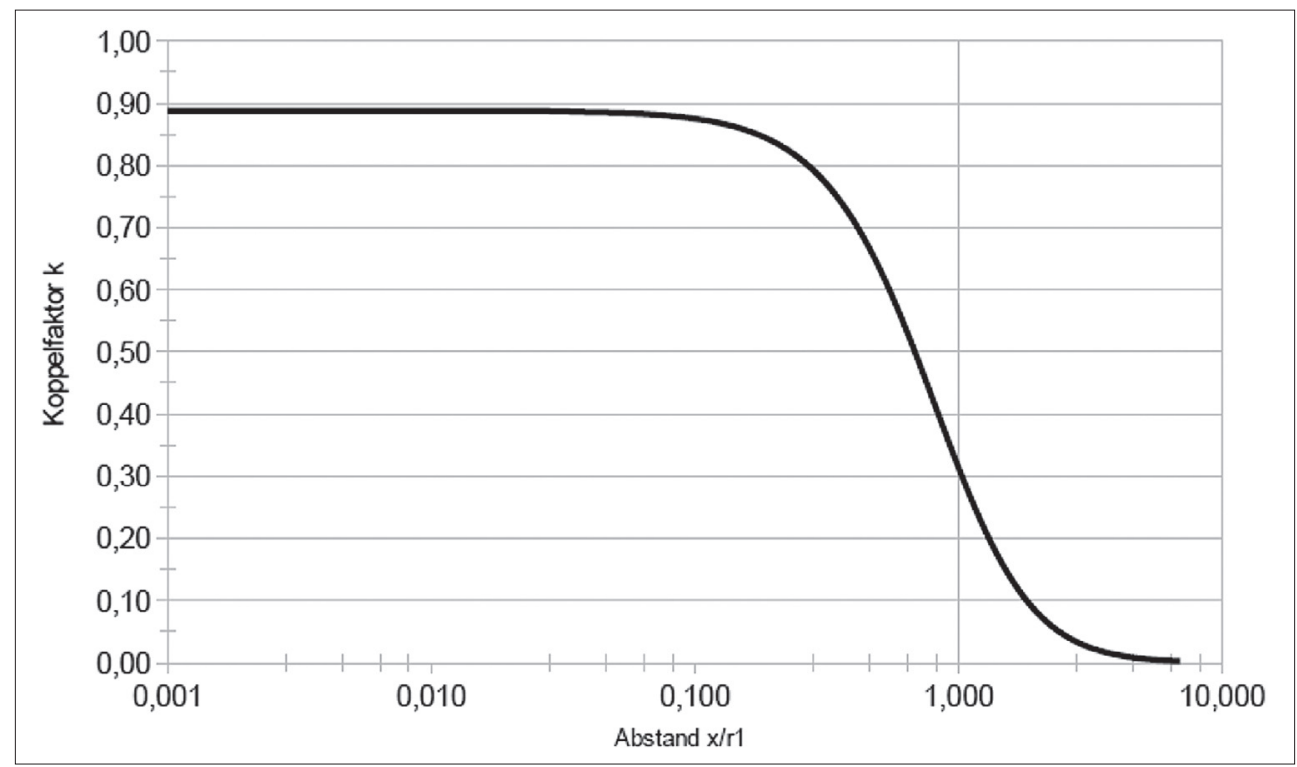

Abb. 2: Verlauf Koppelfaktor, auf Spulenradius normiert

Für die Energieausbeute durch den elektronischen Schaltkreis auf der Sekundärseite ist überdies entscheidend, wie der Chip an die Spule kontaktiert ist. Dies ist auch ein entscheidendes Merkmal für die Qualität der Datenübertragung zwischen Reader und Transponder und umgekehrt. Die Transponderspulen werden auf Resonanz abgestimmt, um eine höhere Energieausbeute zu erzielen. Die Resonanzfrequenz kann durch die Umgebung verstimmt werden, insbesondere durch das Aufbringen der Etiketten auf Medien wie Bücher und CDs. Die Verstimmung der Resonanzfrequenz durch die Umgebung kann, sofern sie bekannt und einigermaßen konstant ist, bei der Herstellung der Transponder berücksichtigt werden. Ein solcher angepasster Transponder hat dann erst bei Anbringung auf das Medium seine optimale Performance. Durch die sehr unterschiedlichen Medien, die in der Praxis zum Einsatz kommen, ist es nicht möglich die Resonanzfrequenz eines RFID Transponder so abzustimmen, dass sie für alle Medien exakt gleich ist. Es ist ein Kompromiss zu wählen, der innerhalb eines definierten Toleranzbandes liegt.

\section{Charakterisierung von RFID Transponder}

Wie beschrieben handelt es sich bei einem RFID System im 13,56 MHz Bereich um ein verkoppeltes System. Deshalb ist es außerordentlich schwierig, die Performance eines Transponders für sich zu bestimmen, da er immer in Wechselwirkung mit dem Reader steht. Je nach Reader, Readerantenne und Umgebung können mit einem RFID Transponder unterschiedliche Reichweiten erzielt werden. Ebenso kann ein Reader mit Transpondern verschiedener Hersteller unterschiedlich gut zusammenarbeiten. Deshalb wird der Transponder in einem genau definierten Readerumfeld charakterisiert. 
Zur Charakterisierung wird nicht die Reichweite herangezogen, sondern spezifische Merkmale der Luftschnittstelle. Dies ist zum einen die Ansprechfeldstärke $H_{\text {min }}$ des Transponders, also die Feldstärke, die minimal vom Reader erzeugt werden muss, um den Transponder zu aktivieren, ihn also mit ausreichend Energie zu versorgen. Zum anderen wird die Amplitude des Antwortsignals $A_{\text {Response }}$, das vom Transponder erzeugt wird an der Empfangsspule des Readers gemessen. Aus diesen beiden Kennwerten eines Transponders lässt sich dann die mit einem gegebenen Reader erzielbare Reichweite berechnen. Es gibt zwei Reichweitenbegrenzungen im System:

1. Bis zu welcher Distanz kann ein Reader die notwendige minimale Ansprechfeldstärke für einen Transponder erzeugen.

2. Aus welcher Distanz ist der Reader noch in der Lage das vom Transponder erzeugte Antwortsignal zu detektieren.

Man überprüft also, ob ein Reader die benötigte Feldstärke bei der gewünschten Reichweite erzeugt und ob die Amplitudenänderungen, die die Transponderantworten am Ausgang der Primärantenne des Readers verursachen auch vom Reader detektiert werden können.

Zur Bestimmung dieser Parameter ist eine definierte Testumgebung notwendig. In internationalen Standards sind solche Setups festgelegt. So sind im Standard ISO10373 Testaufbauten und -methoden für die Charakterisierung von Chipkarten bei 13,56 MHz definiert. Andere RFID Transponderarten werden in ISO18046 und ISO18047 berücksichtigt. Diese Standards definieren auch zu prüfenden Merkmale und deren Grenzwerte. Bisher nicht definiert ist, wie der Einfluss unterschiedlicher Medien berücksichtigt werden kann. Da die Variation der Medien in Größe, Dicke und Beschaffenheit des Materials wie etwa des Einbandes eines Buches sehr groß ist, muss versucht werden einige wenige Referenzmedien festzulegen, die einen Großteil der Medien am Besten repräsentiert. Ausnahmemedien, die nicht vom Referenzmedium abgedeckt werden, wird es aber immer geben.

Um das Verhalten der Transponder noch genauer zu ermitteln, können die Parameter über einen größeren Frequenzbereich gemessen werden. Durch diese Messung erhält man eine Aussage über die Resonanzfrequenz der RFID Transponderantenne. Idealerweise sollte die Resonanzfrequenz des Transponders bei 13,56 MHz liegen, um eine möglichst hohe Reichweite zu erzielen. In der Praxis kann sich aber durch die Umgebung die Resonanzfrequenz verschieben. Dies geschieht insbesondere durch das Medium selber, auf dem das RFID Etikett angebracht wird. Auch die Transponder selber können sich gegenseitig beeinflussen, wenn sie zu nahe aneinander gebracht werden.

Weiterhin spielt die Güte der Spule eine entscheidende Rolle. Je höher die Güte, desto größer die empfangene Energie am Transponder. Mit einer höheren Güte wird aber auch die Bandbreite kleiner, was sich wiederum negativ auf die Amplitude des Antwortsignals des Transponders auswirkt. Die Güte einer Spule wird sowohl vom Aufbau der Spule als auch von der Umgebung also beispielsweise vom Medium beeinflusst. 


\section{Auswirkungen der Medien auf die Transponder}

Die Medien haben Auswirkungen auf die RFID Etiketten, insbesondere wird die Resonanzfrequenz der Transponder durch die Beschaffenheit der Medien verstimmt. Abbildung 3 zeigt exemplarisch Messungen der Resonanzfrequenz verschiedener RFID Etiketten mit und ohne Medium. Es wurde dabei mit 4 verschiedenen Medien gemessen. Es ist deutlich zu erkennen, dass sich die Resonanzfrequenzen durch die Medien nach unten zur nominellen Resonanzfrequenz von 13,56 MHz hin verschieben.

Ohne Medium liegen die Resonanzfrequenzen meist über 13,56 MHz. Die Höhe der Frequenzverschiebung ist bei den verschiedenen Transpondertypen unterschiedlich. Wie das Diagramm ebenfalls zeigt variiert die Frequenzverschiebung auch mit der Art und Beschaffenheit des Mediums.

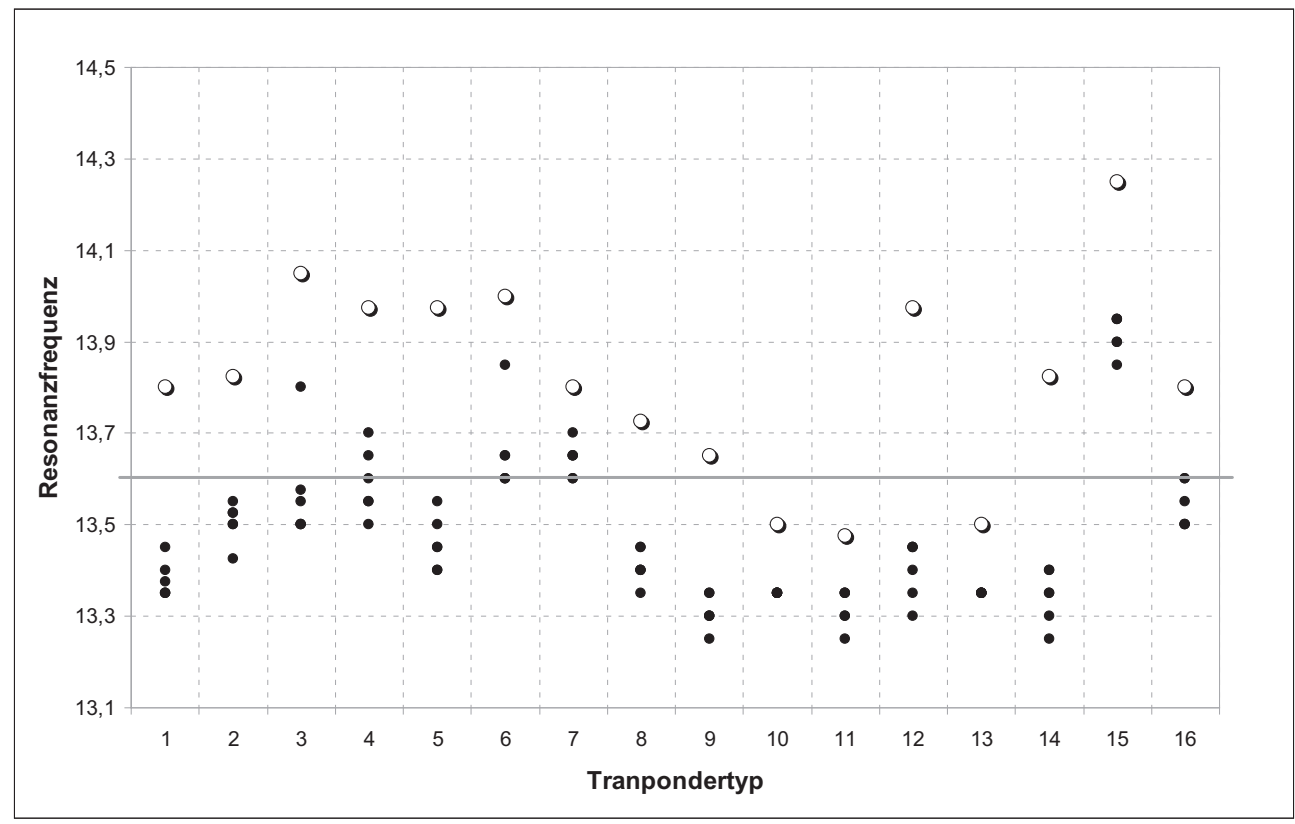

Abb. 3: Messung der Resonanzfrequenz verschiedener Transponder mit verschiedenen Medien (schwarz) und ohne Medium (weiss).

Durch die individuelle Ausgestaltung der RFID Etiketten durch den Hersteller können die Auswirkungen durch die Medien kompensiert werden. Dabei spielt die Größe, Material und Form der Antennenspule auf dem Etikett eine wesentliche Rolle. Es können unterschiedliche Materialen wie Kupfer oder Aluminium verwendet werden. Die Anzahl und der Abstand der Windungen der Spule, sowie die Dicke der Leiterbahnen haben wesentlichen Einfluss auf die Resonanzfrequenz und Güte der Spule. Weiterhin kommen unterschiedliche Chips von verschiedenen Halbleiterherstellern zum Einsatz, die auch unterschiedlich kontaktiert werden können. Dadurch ergibt sich eine Vielzahl von Freiheitsgraden, mit denen ein RFID Etikett individuell an die jeweiligen Anforderungen angepasst werden kann. Entscheidend ist, die Anforderungen der Bibliothek und damit 
im Wesentlichen die Auswirkungen verschiedener Medien auf das RFID Etikett korrekt zu bestimmen. Nur so ist eine bessere Auswahl oder Optimierung der RFID Etiketten für diese Anwendung möglich.

\section{Zusammenfassung und Ausblick}

Dieser Beitrag versucht auf das Thema Qualitätsstandards bei RFID Etiketten für den Einsatz in Bibliotheken einzugehen. Qualifizierungsnormen für HF-RFID Transponder bei 13,56 MHz existieren, müssen aber für die Anwendung im Bibliotheksbereich angepasst werden. Verbindliche Grenzwerte für minimale Ansprechfeldstärke, Amplitude des Transponder-Antwortsignals und der daraus resultierenden Reichweite auf dem Medium müssen für die Anwendung bei Bibliotheken und auf Medien noch festgelegt werden. Der dafür ins Leben gerufene runde Tisch zur Sicherung der Qualität von RFID Etiketten im Bibliothekswesen arbeitet mit den Herstellern von RFID Etiketten und Chips an einem verbindlichen Regelwerk für die Ausschreibung von RFID Etiketten.

\section{Literatur und Internetquellen}

[1] Finkenzeller, Klaus (2002): RFID Handbuch, 3. Auflage. Hansen Verlag München Wien, ISBN 3-446-22071-2

[2] ISO/IEC 10373: Identification Cards - Test methods

[3] ISO/IEC 18046 (2007): Information technology - Automatic identification and data capture techniques - Radio frequency identification device performance test methods

[4] ISO/IEC TR 18047-3 (2008): Information technology -Radio frequency identification device conformance test methods - Part 3: Test methods for air interface communications at $13,56 \mathrm{MHz}$ 